Chapman University

Chapman University Digital Commons

Mathematics, Physics, and Computer Science

Science and Technology Faculty Articles and

Faculty Articles and Research

Research

$1-6-2017$

Experimental Demonstration of Direct Path State Characterization by Strongly Measuring Weak Values in a Matter-Wave Interferometer

Tobias Denkmayr

AtomInstitut

Hermann Geppert

AtomInstitut

Hartmut Lemmel

AtomInstitut

Mordecai Waegell

AtomInstitut

Justin Dressel

Chapman University,dressel@chapman.edu

See next page for additional authors

Follow this and additional works at: http://digitalcommons.chapman.edu/scs_articles

Part of the Quantum Physics Commons

Recommended Citation

T. Denkmayr, H. Geppert, H. Lemmel, M. Waegell, J. Dressel, Y. Hasegawa, and S. Sponar, Phys. Rev. Lett. 118, 010402 (2017).

This Article is brought to you for free and open access by the Science and Technology Faculty Articles and Research at Chapman University Digital Commons. It has been accepted for inclusion in Mathematics, Physics, and Computer Science Faculty Articles and Research by an authorized administrator of Chapman University Digital Commons. For more information, please contact laughtin@chapman.edu. 


\section{Experimental Demonstration of Direct Path State Characterization by Strongly Measuring Weak Values in a Matter-Wave Interferometer}

\section{Comments}

This article was originally published in Physical Review Letters, volume 118, in 2017. DOI:10.1103/

PhysRevLett.118.010402

\section{Copyright}

American Physical Society

\section{Authors}

Tobias Denkmayr, Hermann Geppert, Hartmut Lemmel, Mordecai Waegell, Justin Dressel, Yuji Hasegawa, and Stephan Sponar 


\title{
Experimental Demonstration of Direct Path State Characterization by Strongly Measuring Weak Values in a Matter-Wave Interferometer
}

\author{
Tobias Denkmayr, ${ }^{1, *}$ Hermann Geppert, ${ }^{1}$ Hartmut Lemmel, ${ }^{1,2}$ Mordecai Waegell, ${ }^{3}$ Justin Dressel,,${ }^{3,4}$ \\ Yuji Hasegawa, ${ }^{1}$ and Stephan Sponar, ${ }^{1, \dagger}$ \\ ${ }^{1}$ AtomInstitut, TU Wien, Stadionallee 2, 1020 Vienna, Austria \\ ${ }^{2}$ Institut Laue-Langevin, 6, Rue Jules Horowitz, 38042 Grenoble Cedex 9, France \\ ${ }^{3}$ Institute for Quantum Studies, Chapman University, Orange, California 92866, USA \\ ${ }^{4}$ Schmid College of Science and Technology, Chapman University, Orange, California 92866, USA
}

(Received 15 April 2016; published 6 January 2017)

\begin{abstract}
A method was recently proposed and experimentally realized for characterizing a quantum state by directly measuring its complex probability amplitudes in a particular basis using so-called weak values. Recently, Vallone and Dequal [Phys. Rev. Lett. 116, 040502 (2016)] showed theoretically that weak measurements are not a necessary condition to determine the weak value. Here, we report a measurement scheme used in a matter-wave interferometric experiment in which the neutron path system's quantum state was characterized via direct measurements, using both strong and weak interactions. Experimental evidence is given that strong interactions outperform weak ones for tomographic accuracy. Our results are not limited to neutron interferometry, but can be used in a wide range of quantum systems.
\end{abstract}

DOI: 10.1103/PhysRevLett.118.010402

Ever since Aharonov, Albert, and Vaidman (AAV) introduced the weak value as "a new kind of value for a quantum variable" [1], it has been a topic of intense debate $[2,3]$. As a generalized form of conditioned observable average [4], it has offered new insights into quantum paradoxes, such as Hardy's paradox [5-7], the three-box paradox [8,9], and the quantum Cheshire cat [10,11], which in turn have fueled ongoing discussions about its physical interpretation [12-16]. Independent of interpretation, however, the weak value is experimentally pragmatic [17], finding uses in high precision metrology [18-22] and state characterization [23-26].

This latter state characterization constructs complex quantum state amplitudes in a particular basis directly from ratios of averaged detector outputs. The simplicity of this protocol is well-suited for high-dimensional state reconstructions, such as for the 19200-dimensional state, sampled using compressive sensing in Ref. [26]. The weak value is an experimentally accessible complex number,

$$
\langle\hat{\mathrm{A}}\rangle_{w}=\frac{\left\langle\psi_{f}|\hat{\mathrm{A}}| \psi_{i}\right\rangle}{\left\langle\psi_{f} \mid \psi_{i}\right\rangle}
$$

where $\hat{\mathrm{A}}$ is an observable of interest, while $\left|\psi_{i}\right\rangle$ and $\left|\psi_{f}\right\rangle$ are initial (preselected) and final (postselected) system states, respectively. To measure such weak values, an ancillary probe must be coupled to the system of interest. Traditionally, this coupling has been kept minimally disturbing (weak); however, several theory works have recently noted that the weakness of the interaction is not necessary [27-29] and may even be a detriment to the accuracy of the state reconstruction [30]. We investigate these claims in this Letter, and experimentally confirm that stronger interaction strengths improve the accuracy of direct state characterization, using weak values.

Originally, AAV constructed the weak value formalism in a nonrelativistic quantum framework and hence, it should be first and foremost applicable to massive quantum systems [1]. In general, optical experiments with matter waves provide excellent conditions to demonstrate the peculiarities of quantum mechanics [31-33]. However, because of the small coherence volume of massive particle beams, an experimental demonstration of a weak value's measurement in a simple massive-particle system proved to be difficult; the first experimental determination of a weak value was realized in a purely optical setup [34]. Significant improvements in the technique of neutron interferometry [35] made it possible to fully determine the weak value of a neutron's spin operator with high precision $[36,37]$. Neutron interferometry has been established as a powerful experimental method to investigate the foundations of quantum mechanics [38-43]. In combination with the weak value measurement scheme, it offers an experimental window into previously inaccessible parts of massive quantum systems.

Here, we present an experiment in which the neutron's path degree of freedom's (DOF) [44] state vector is characterized using weak values. The observable of interest is the Pauli operator $\hat{\sigma}_{z}^{p}$, and the neutron's spin DOF serves as a meter system. The measurement of $\left\langle\hat{\sigma}_{z}^{p}\right\rangle_{w}$ makes it possible to directly characterize the preselected path state. The weak values are obtained through weak and strong interactions, and the precision (as well as the accuracy) of both experimental approaches are quantified. The experimental results support the statements made in $[28,30]$ that stronger measurements indeed outperform weak ones. 
The measurement scheme starts with the initial state

$\left|\Psi_{i}\right\rangle=\left|P_{i}\right\rangle\left|S_{i}\right\rangle=\left(c_{+}\left|P_{z} ;+\right\rangle+c_{-}\left|P_{z} ;-\right\rangle\right)\left|S_{x} ;+\right\rangle$,

where $\left|P_{i}\right\rangle$ is the initial path and $\left|S_{i}\right\rangle$, the initial spin state. $\left|P_{z} ;+\right\rangle$ and $\left|P_{z} ;-\right\rangle$ are the eigenstates of path I and II, respectively, with the corresponding probability amplitudes $c_{+}$and $c_{-} .\left|S_{x} ;+\right\rangle$ denotes a spin state that is aligned along the positive $x$ axis. A general form for a preselected path state is given by

$$
\left|P_{i}\right\rangle=\cos \left(\frac{\theta}{2}\right)\left|P_{z} ;+\right\rangle+\exp (i \phi) \sin \left(\frac{\theta}{2}\right)\left|P_{z} ;-\right\rangle,
$$

where $\phi$ represents the relative phase and $\theta$, the weight of the two eigenstates. The probability amplitudes $c_{+}$and $c_{-}$ are linked to the general state by $\phi=\arg \left(c_{+}\right)-\arg \left(c_{-}\right)$ and $\cos \theta=\left|c_{+}\right|^{2}-\left|c_{-}\right|^{2}$.

Equation (2) describes a completely separable state. There is no coupling between the spin and path DOF. As a next step, a coupling is created by a unitary evolution consisting of path-dependent spin rotations. More precisely, the spin is rotated by a certain angle $\alpha$ around the $z$ axis in the $x y$ plane, with positive (clockwise) rotations in path I and negative (counter clockwise) ones in path II. The interaction Hamiltonian for such a measurement is $\hat{H}_{\text {int }}=-\vec{\mu} \cdot \vec{B} \hat{\Pi}_{z+}^{p}+\vec{\mu} \cdot \vec{B} \hat{\Pi}_{z-}^{p}$, where $\hat{\Pi}_{z \pm}^{p}$ are the projection operators on the path eigenstates $\left|P_{z} ;+\right\rangle$ and $\left|P_{z} ;-\right\rangle, \vec{\mu}$ is the neutron's magnetic moment, and $\vec{B}=\left(0,0, B_{z}\right)$, an applied magnetic field.

The action of $\hat{H}_{\text {int }}$ on the composite system $\left|\Psi_{i}\right\rangle$ is described by an evolution operator $\left|\Psi^{\prime}\right\rangle=e^{-i / \hbar \int \hat{H}_{\text {int }} d t}\left|\Psi_{i}\right\rangle=$ $e^{-i \alpha \hat{\sigma}_{z}^{s} \hat{\sigma}_{z}^{p} / 2}\left|\Psi_{i}\right\rangle$. The angle of rotation $\alpha$ is given by $-2 \mu \mathrm{B}_{z} \tau / \hbar$, where $\tau$ is the neutron's transit time in the magnetic field region. $\alpha$ is the relevant parameter for the interaction strength of the measurement. $\hat{\sigma}_{z}^{s}$ is the generator of spin rotations around the $z$ axis. The Pauli operator $\hat{\sigma}_{z}^{p}$ is given by $\hat{\sigma}_{z}^{p}=\left|P_{z} ;+\right\rangle\left\langle P_{z} ;+|-| P_{z} ;-\right\rangle\left\langle P_{z} ;-\right|$.

In the standard weak measurement procedure [36], the evolution operator $\exp \left(-i \alpha \hat{\sigma}_{z}^{s} \hat{\sigma}_{z}^{p} / 2\right)$ is series expanded around $\alpha$ and by neglecting higher orders of $\alpha$, an approximation for $\alpha \ll 1$ is made. Here, however, the analytical relation $\exp \left(-i \alpha \hat{\sigma}_{z}^{s} \hat{\sigma}_{z}^{p} / 2\right)=\cos (\alpha / 2)-$ $i \hat{\sigma}_{z}^{s} \hat{\sigma}_{z}^{p} \sin (\alpha / 2)$ is used [28]. No approximation is made. Therefore, the calculations hold for arbitrary interaction strengths. The analytic form of the state after the interaction is given by

$$
\left|\Psi^{\prime}\right\rangle=\cos \left(\frac{\alpha}{2}\right)\left|P_{i}\right\rangle\left|S_{x} ;+\right\rangle-i \hat{\sigma}_{z}^{p} \sin \left(\frac{\alpha}{2}\right)\left|P_{i}\right\rangle\left|S_{x} ;-\right\rangle .
$$

The final step of the measurement scheme is the postselection. The path is postselected on the final state

$$
\left|P_{f}\right\rangle=\left|P_{x} ;+\right\rangle=\frac{1}{\sqrt{2}}\left(\left|P_{z} ;+\right\rangle+\left|P_{z} ;-\right\rangle\right) .
$$

The action of the path postselection is equivalent to a projection onto $\left|P_{f}\right\rangle\left\langle P_{f}\right|$. It leads to final state $\left|\Psi_{f}\right\rangle$, which has the form

$$
\begin{aligned}
\left|\Psi_{f}\right\rangle= & \left\langle P_{f} \mid P_{i}\right\rangle\left[\cos \left(\frac{\alpha}{2}\right)\left|S_{x} ;+\right\rangle\right. \\
& \left.-\mathrm{i} \sin \left(\frac{\alpha}{2}\right)\left\langle\hat{\sigma}_{z}^{p}\right\rangle_{w}\left|S_{x} ;-\right\rangle\right]\left|P_{f}\right\rangle .
\end{aligned}
$$

Finally, the weak value of $\hat{\sigma}_{z}^{p}$ is determined by evaluating the pointer system. Projective measurements along the six spin directions $\pm x, \pm y$, and $\pm z$, yield six intensities $I_{j \pm}=$ $\left|\left\langle S_{j} ; \pm \mid \Psi_{f}\right\rangle\right|^{2}$ with $(j=x, y, z)$, which allow us to extract the imaginary and real parts, as well as the modulus of the path operator's weak value. It is straightforward to derive the relations

$$
\begin{aligned}
\operatorname{Re}\left(\left\langle\hat{\sigma}_{z}^{p}\right\rangle_{w}\right) & =\frac{1}{2} \cot \left(\frac{\alpha}{2}\right) \frac{I_{y+}-I_{y-}}{I_{x+}} \\
\operatorname{Im}\left(\left\langle\hat{\sigma}_{z}^{p}\right\rangle_{w}\right) & =\frac{1}{2} \cot \left(\frac{\alpha}{2}\right) \frac{I_{z+}-I_{z-}}{I_{x+}} \\
\left|\left\langle\hat{\sigma}_{z}^{p}\right\rangle_{w}\right| & =\cot \left(\frac{\alpha}{2}\right) \sqrt{\frac{I_{x-}}{I_{x+}}}
\end{aligned}
$$

which connect the intensities $I_{j \pm}$ to all components of $\left\langle\hat{\sigma}_{z}^{p}\right\rangle_{w}$. It has to be stressed that no approximations are made to derive this result: relations ( $7 \mathrm{a})$ to (7c) hold for any value of $\alpha$, i.e., for arbitrary measurement strengths. The above argument is not limited to the neutron's spin and path DOF, but it can be applied to any coupling between two two-level quantum systems.

Since $\left\langle\hat{\Pi}_{z \pm}^{p}\right\rangle_{w}=\mathbb{1} \pm\left\langle\hat{\sigma}_{z}^{p}\right\rangle_{w} / 2$, the complete determination of the weak value of the Pauli operator $\hat{\sigma}_{z}^{p}$ also gives the weak values of the projection operators on each path eigenstate. These, in turn, characterize the measured preselected path state [24]

$$
\left|P_{i}^{m}\right\rangle=\frac{\left\langle\hat{\Pi}_{z+}^{p}\right\rangle_{w}\left|P_{z} ;+\right\rangle+\left\langle\hat{\Pi}_{z-}^{p}\right\rangle_{w}\left|P_{z} ;-\right\rangle}{\sqrt{\left|\left\langle\hat{\Pi}_{z+}^{p}\right\rangle_{w}\right|^{2}+\left|\left\langle\hat{\Pi}_{z-}^{p}\right\rangle_{w}\right|^{2}}} .
$$

By denoting the normalization factor as $\nu \equiv$ $1 / \sqrt{\left|\left\langle\hat{\Pi}_{z+}^{p}\right\rangle_{w}\right|^{2}+\left|\left\langle\hat{\Pi}_{z-}^{p}\right\rangle_{w}\right|^{2}}$, the probability amplitudes of $\left|P_{i}^{m}\right\rangle$ are given by $c_{+}=\nu\left\langle\hat{\Pi}_{z+}^{p}\right\rangle_{w}$ and $c_{-}=\nu\left\langle\hat{\Pi}_{z-}^{p}\right\rangle_{w}$. They are directly proportional to quantities that are determined experimentally, namely to the path projection operators' weak values. Using the weak value's definition given by Eq. (1), as well as the pre- and postselected path states of Eqs. (3) and (5), respectively, one expects $\left\langle\hat{\Pi}_{z \pm}^{p}\right\rangle_{w}$ to be

$$
\left\langle\hat{\Pi}_{z \pm}^{p}\right\rangle_{w}=\frac{\left\langle P_{f}\left|\hat{\Pi}_{z \pm}^{p}\right| P_{i}\right\rangle}{\left\langle P_{f} \mid P_{i}\right\rangle}=\frac{1}{2} \mp \frac{i}{2} \tan \left(\frac{\phi}{2}\right),
$$

if one assumes that $\theta=\pi / 2$ in Eq. (3), as is the case for a 50:50 beam splitter of a Mach-Zehnder type interferometer.

A neutron interferometric experiment was performed at the beam line S18 at the high-flux research reactor at the Institut Laue-Langevin (ILL) in Grenoble, France. A 


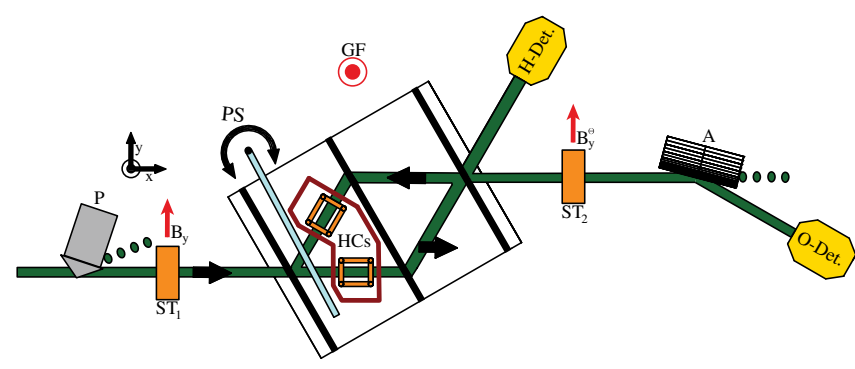

FIG. 1. Schematic drawing of the neutron interferometer setup implementing spin polarization.

schematic drawing of the interferometric setup is depicted in Fig. 1.

From a white neutron, beam particles with a wavelength $\lambda_{0}=1.91 \AA\left(\Delta \lambda / \lambda_{0} \sim 0.02\right)$ are selected by a triple-bounce silicon perfect-crystal monochromator and subsequently, pass magnetic prisms $(\mathrm{P})$. They deflect the spin-down component out of the Bragg condition of the interferometer crystal, such that only spin-up neutrons are accepted by the interferometer [45]. The prisms polarize the neutron beam along the positive $z$ axis. For our experiment, the degree of polarization was determined to be over $99 \%$. A DC coil $\left(\mathrm{ST}_{1}\right)$ turns the neutron spin by $\pi / 2$ due to Larmor precession within the coil, so that the spin is aligned along the positive $x$ axis. To tune the relative phase $\chi$, a parallelsided sapphire slab is inserted between the first and the second plate of the interferometer as a phase shifter (PS).

After the phase shifter, the initial state $\left|\Psi_{i}\right\rangle$ is generated, and the preselection procedure is complete. Inside the interferometer, a coil in Helmholtz configuration (HC) in each beam path enables us to perform path-dependent spin rotations, coupling the path and spin DOF [35]. In addition to the external guide field (GF), which points in the $+z$ direction and is applied around the whole setup, the coils produce extra magnetic fields in the $\pm z$ direction that cause the neutron spins' Larmor precession frequency $\omega_{L}$ to decrease or increase, depending on the sign of the field. The strength of the magnetic field determines the magnitude of the rotation angle $\alpha$. The experiment is performed with two different values of $\alpha$. To test the interaction in a weak regime, $\alpha$ is set to $15^{\circ}$. For the strong interactions, $\alpha$ is set to $90^{\circ}$, which corresponds to the maximum measurement strength due to the orthogonality of the spin states after the interaction.

At the interferometer's third plate, the beams are recombined and the path postselection is carried out. Only neutrons leaving the interferometer in the forward direction (O-Det.) are spin analyzed. The spin analysis is performed by a second DC coil $\left(\mathrm{ST}_{2}\right)$, mounted on a translation stage in combination with a CoTi supermirror analyzer $(A)$, which acts as a filter that lets only the $\left|S_{z} ;+\right\rangle$ spin state pass. The second DC coil can be moved in the $\pm x$ direction. Finally, the neutrons are detected by a ${ }^{3} \mathrm{He}$ counter.

The intensity modulations $I_{j \pm}$ of both experimental runs are depicted in Fig. 2. The three left panels show the interferograms of the weak interaction $\left(\alpha=15^{\circ}\right)$. For $I_{x+}^{w}$, both the pre- and postselected spin state are $\left|S_{x} ;+\right\rangle$, leading to a large count rate. In contrast, the count rate of $I_{x-}^{w}$ is very low due to the orthogonality of initial and final spin states. $I_{y \pm}^{w}$ are identical and have on average, half of the maximal count rate. $I_{z \pm}^{w}$ are phase shifted to each other by two times $\alpha$, and they show the same average count rate. In the three right panels, the interferograms for the strong interaction are shown. Because of the large spin rotation of $\alpha= \pm 90^{\circ}$ in each beam path, $I_{x \pm}^{\text {st }}$ now show the same average count rate, while being phase shifted by $\pi . I_{y \pm}^{\text {st }}$ show only negligibly little contrast. The phase shift between $I_{z+}^{\text {st }}$ and $I_{z-}^{\text {st }}$ is now also expected to be $\pi$ and easy to resolve. The data are fitted with a cosine function of the form $g(\chi)=a+$ $b \cos (\bar{c} \chi+d)$, where the free fit parameters $a, b$, and $d$ are the average, the amplitude, and the phase of the interferogram, respectively. The average period $\bar{c}$ is obtained from the $H$-detector data of all interferograms and held fixed.

Some advantages of the strong measurement approach can already be seen in the differences between the interference fringes of the weak and the strong interaction measurements. To extract the imaginary part of $\left\langle\hat{\sigma}_{z}^{p}\right\rangle_{w}$, the intensities $I_{z \pm}$ and $I_{x+}$ are used. While $I_{x+}$ acts as a normalization factor, resolving the phase shift between $I_{z+}$ and $I_{z-}$ is crucial to determine $\operatorname{Im}\left(\left\langle\hat{\sigma}_{z}^{p}\right\rangle_{w}\right)$. Since this phase shift is expected to be two times $\alpha$, it is much harder to resolve in the weak interaction case. Similarly, $\operatorname{Re}\left(\left\langle\hat{\sigma}_{z}^{p}\right\rangle_{w}\right)$ is extracted from the intensities $I_{y \pm}$ and $I_{x+}$. Because the weak value's real part is expected to be zero, $I_{y+}$ and $I_{y-}$ are

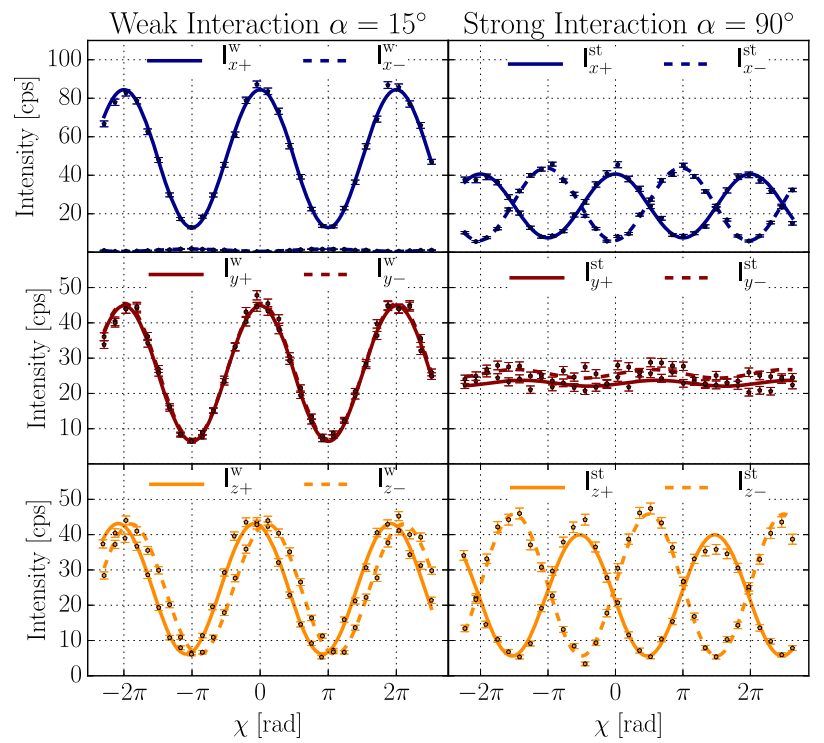

FIG. 2. Interference fringes of the weak measurement $\left(\alpha=15^{\circ}\right)$ on the left side and those of the strong measurement $\left(\alpha=90^{\circ}\right)$ on the right side. The directions of spin analysis $\pm x, \pm y$, and $\pm z$ are shown in the top, middle, and bottom rows, respectively. The solid and dashed lines show the least square fits for the + andanalysis directions, respectively. The error bars show one standard deviation. The background has already been subtracted from all interferograms. 
very close to equal [36]. Furthermore, $I_{y \pm}$ loses contrast for larger $\alpha$ due to the spin rotation inside the interferometer, leading to an orthogonal spin state. For $\alpha=90^{\circ}$, the spin state is completely orthogonal and shows no contrast.

Finally, the modulus of $\left\langle\hat{\sigma}_{z}^{p}\right\rangle_{w}$ is directly obtained from the $I_{x \pm}$ data. It is proportional to the square root of $I_{x-} / I_{x+}$. The advantages of the strong interaction approach are intuitively understood. Here, the discrimination of the relevant signal from the background is crucial: For $\alpha=0^{\circ}, I_{x-}$ is also expected to be zero and the signal becomes larger with increasing $\alpha$. If $\alpha$ is kept small, as in the weak measurement approach, it is very hard to discriminate the intensity from the background.

The intensities recorded in the experiment, completely determine $\left\langle\hat{\sigma}_{z}^{p}\right\rangle_{w}$. Using $\left\langle\hat{\sigma}_{z}^{p}\right\rangle_{w}$, the weak values of the path projection operators $\left\langle\hat{\Pi}_{z+}^{p}\right\rangle_{w}$ and $\left\langle\hat{\Pi}_{z-}^{p}\right\rangle_{w}$ are calculated. They, in turn, are directly related to the preselected path state, making $\left|P_{i}^{m}\right\rangle$ available. The results of such a direct state characterization are shown in Fig. 3, where the weak and the strong interaction approaches are compared to each other. The upper panels show the normalization factor $\nu$, which connects the probability amplitudes $c_{+}$and $c_{-}$to $\left\langle\hat{\Pi}_{z \pm}^{p}\right\rangle_{w}$ for both the weak and the strong interaction cases. The middle panels depict the parameter, describing the relative weighting $\theta$ of the general preselected state described by Eq. (3) again for both measurement approaches. Finally, the relative phase $\phi$ is depicted in the lower panels. The interferograms' individual points in Fig. 2 directly map to the points in Fig. 3, when using Eqs. (7a)-(7c). The lines represent the theoretical prediction based upon the calibration of the experimental setup. They take into account the period $\bar{c}$ obtained from the $H$-detector

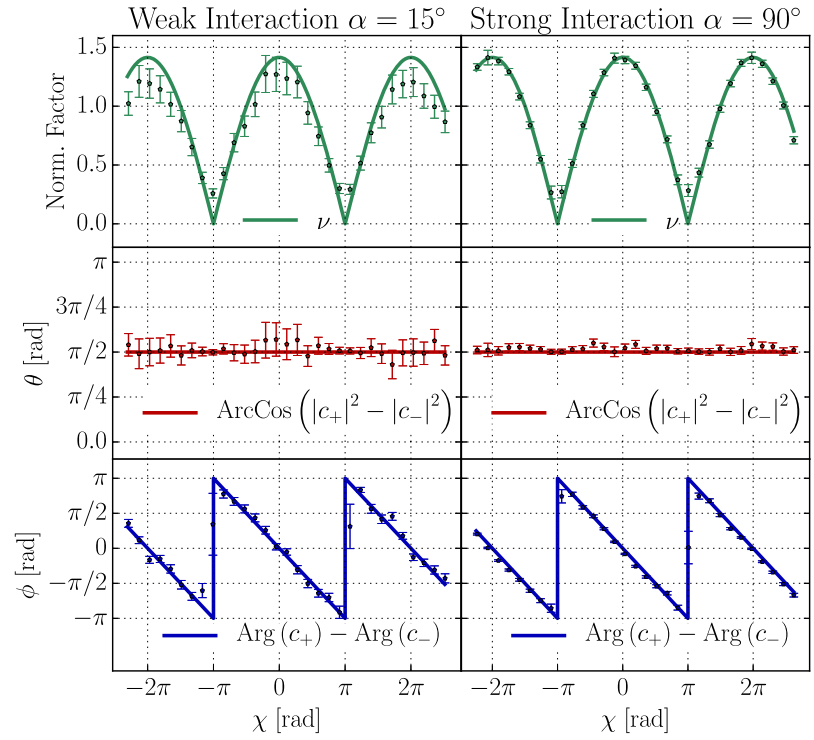

FIG. 3. Measurement results for the path state vector for both the weak $\left(\alpha=15^{\circ}\right)$ and the strong measurement $\left(\alpha=90^{\circ}\right)$ cases: The error bars show one standard deviation. The solid lines are the theoretical predictions. data and the phase $d$ of the $I_{x+}$ intensity. In addition, the contrast of the interferometer has been considered for the state characterization. In the final results, the advantages of the strong interaction approach are evident.

While both measurements are in good agreement with the theoretical predictions, the strong measurement results are significantly better, in terms of precision $\bar{\sigma}$ (a measure of fluctuation) and accuracy $\bar{\Delta}$ (a measure of deviation from the theoretical prediction).

For the evaluation of precision of weak and strong interaction approaches, we use the root mean square statistical error

$$
\bar{\sigma}=\sqrt{\frac{1}{N} \sum_{i}^{N}\left|\sigma_{i}\right|^{2} .}
$$

The root mean square deviation

$$
\bar{\Delta}=\sqrt{\frac{1}{N} \sum_{i}^{N}\left|t_{i}-m_{i}\right|^{2}}
$$

of each measured point $m_{i}$ from the theoretical predictions $t_{i}$, in turn, is a measure of accuracy. In Table I, the results of this quantitative analysis are listed.

The strong interaction scheme surpasses the weak one in both accuracy and precision for each and every one of the experimentally determined parameters. For all measured quantities, $\bar{\sigma}$ is roughly twice as large in the weak interaction case. Also, the mean deviation from the theoretical predictions is smaller for the strong interaction approach. There is another important experimental factor that has to be taken into account: the measurement time. To resolve the small phase shifts between $I_{z+}$ and $I_{z-}$, as well as to discern $I_{x-}$ from the background, long counting times were necessary for the weak interaction. If the signal is not clearly distinguished from the background, nonsensical results are obtained, making it impossible to quantify the accuracy of the measurement. Therefore, for each point on the weak interaction curve, a counting time of 540 seconds was necessary, while 290 seconds were sufficient for the strong one. To make the precision values in Table I more directly comparable, it is possible to scale the error bars of the weak interaction data. Doing so confirms that the precision scales with the square root of the measurement; i.e., the weak interaction results become even worse by a

TABLE I. Quantitative comparison of precision $\bar{\sigma}$ and accuracy $\bar{\Delta}$, as defined in Eqs. (10) and (11), of the weak and the strong

\begin{tabular}{|c|c|c|c|c|c|}
\hline \multicolumn{3}{|c|}{ Precision $\bar{\sigma}$} & \multicolumn{3}{|c|}{ Accuracy $\bar{\Delta}$} \\
\hline & Weak & Strong & & Weak & Strong \\
\hline$\nu$ & 0.100 & 0.036 & $\nu$ & 0.152 & 0.062 \\
\hline$\theta$ & 0.191 & 0.065 & $\theta$ & 0.100 & 0.067 \\
\hline$\phi$ & 0.355 & 0.159 & $\phi$ & 0.860 & 0.580 \\
\hline
\end{tabular}
interaction approaches. 
factor of $\sim \sqrt{1.86}$. This is because of the Poissonian counting statistics of the recorded intensities.

Our weak value determination protocol makes it possible to obtain weak values of a two-level quantum system with high accuracy and arbitrary measurement strengths. Increasing the measurement strength in our scheme provides a clear discrimination of small signals from the background. This is particularly significant whenever dealing with low intensities. Our measurement scheme is not limited to the neutron spin and path DOF, but is in fact, completely general and can be used for any coupling between two two-level quantum systems. Furthermore, as long as the meter system is two dimensional, our measurement scheme can be slightly modified and used to determine projection operator's weak values of any discrete $n$-dimensional quantum system.

In summary, we have presented a weak value determination scheme via arbitrary interaction strengths. We have applied it to experimentally determine weak values using both weak and strong interactions. We have directly characterized the preselected state of the investigated quantum system, including its normalization factor $\nu$, its relative phase $\phi$, and the weight of its eigenstates $\theta$. Experimental evidence is given that strong interactions are superior in terms of accuracy and precision, as well as required measurement time.

We acknowledge support by the Austrian Science Fund (FWF) Project No. P24973-N20 \& No. P25795-N20. In addition to that, we thank the ILL for its hospitality and continuous support. This research was supported (in part) by the Fetzer-Franklin Fund of the John E. Fetzer Memorial Trust. Helpful discussions with Prof. M. Suda from the Austrian Institute of Technology are kindly acknowledged.

*tdenkmayr@ati.ac.at †sponar@ati.ac.at

[1] Y. Aharonov, D. Z. Albert, and L. Vaidman, Phys. Rev. Lett. 60, 1351 (1988).

[2] I. M. Duck, P. M. Stevenson, and E. C. G. Sudarshan, Phys. Rev. D 40, 2112 (1989).

[3] A. J. Leggett, Phys. Rev. Lett. 62, 2325 (1989).

[4] L. M. Johansen, Phys. Lett. A 322, 298 (2004).

[5] L. Hardy, Phys. Rev. Lett. 68, 2981 (1992).

[6] J. S. Lundeen and A. M. Steinberg, Phys. Rev. Lett. 102, 020404 (2009).

[7] K. Yokota, T. Yamamoto, M. Koashi, and N. Imoto, New J. Phys. 11, 033011 (2009).

[8] Y. Aharonov and L. Vaidman, J. Phys. A 24, 2315 (1991).

[9] K. J. Resch, J. S. Lundeen, and A. M. Steinberg, Phys. Lett. A 324, 125 (2004).

[10] Y. Aharonov, S. Popescu, D. Rohrlich, and P. Skrzypczyk, New J. Phys. 15, 113018 (2013).

[11] T. Denkmayr, H. Geppert, S. Sponar, H. Lemmel, A. Matzkin, J. Tollaksen, and Y. Hasegawa, Nat. Commun. 5, 4492 (2014).

[12] B. E. Y. Svensson, Quanta 2, 18 (2013).

[13] C. Ferrie and J. Combes, Phys. Rev. Lett. 113, 120404 (2014).
[14] D. Sokolovski, Phys. Lett. A 379, 1097 (2015).

[15] M. F. Pusey, Phys. Rev. Lett. 113, 200401 (2014).

[16] J. Dressel, Phys. Rev. A 91, 032116 (2015).

[17] J. Dressel, M. Malik, F. M. Miatto, A. N. Jordan, and R. W. Boyd, Rev. Mod. Phys. 86, 307 (2014).

[18] O. Hosten and P. Kwiat, Science 319, 787 (2008).

[19] P. B. Dixon, D. J. Starling, A. N. Jordan, and J. C. Howell, Phys. Rev. Lett. 102, 173601 (2009).

[20] O. S. Magaña-Loaiza, M. Mirhosseini, B. Rodenburg, and R. W. Boyd, Phys. Rev. Lett. 112, 200401 (2014).

[21] G. I. Viza, J. Martínez-Rincón, G. B. Alves, A. N. Jordan, and J. C. Howell, Phys. Rev. A 92, 032127 (2015).

[22] K. Lyons, S. Pang, P. G. Kwiat, and A. N. Jordan, Phys. Rev. A 93, 043841 (2016).

[23] J. S. Lundeen, B. Sutherland, A. Patel, C. Stewart, and C. Bamber, Nature (London) 474, 188 (2011).

[24] J. Z. Salvail, M. Agnew, A. S. Johnson, E. Bolduc, J. Leach, and R. W. Boyd, Nat. Photonics 7, 316 (2013).

[25] M. Malik, M. Mirhosseini, M. P. J. Lavery, J. Leach, M. J. Padgett, and R. W. Boyd, Nat. Commun. 5, 3115 (2014).

[26] M. Mirhosseini, O. S. Magaña-Loaiza, S. M. Hashemi Rafsanjani, and R.W. Boyd, Phys. Rev. Lett. 113, 090402 (2014).

[27] L. M. Johansen, Phys. Lett. A 366, 374 (2007).

[28] G. Vallone and D. Dequal, Phys. Rev. Lett. 116, 040502 (2016).

[29] Y.-X. Zhang, S. Wu, and Z.-B. Chen, Phys. Rev. A 93, 032128 (2016).

[30] J. A. Gross, N. Dangniam, C. Ferrie, and C. M. Caves, Phys. Rev. A 92, 062133 (2015).

[31] H. Rauch and S. A. Werner, Neutron Interferometry (Clarendon, Oxford, 2000).

[32] A. D. Cronin, J. Schmiedmayer, and D. E. Pritchard, Rev. Mod. Phys. 81, 1051 (2009).

[33] M. Arndt, A. Ekers, W. von Klitzing, and H. Ulbricht, New J. Phys. 14, 125006 (2012).

[34] N. W. M. Ritchie, J. G. Story, and R. G. Hulet, Phys. Rev. Lett. 66, 1107 (1991).

[35] H. Geppert, T. Denkmayr, S. Sponar, H. Lemmel, and Y. Hasegawa, Nucl. Instrum. Methods Phys. Res., Sect. A 763, 417 (2014).

[36] S. Sponar, T. Denkmayr, H. Geppert, H. Lemmel, A. Matzkin, J. Tollaksen, and Y. Hasegawa, Phys. Rev. A 92, 062121 (2015).

[37] S. Sponar, T. Denkmayr, H. Geppert, and Y. Hasegawa, Atoms 4, 11 (2016).

[38] H. Rauch, H. Lemmel, M. Baron, and R. Loidl, Nature (London) 417, 630 (2002).

[39] Y. Hasegawa, R. Loidl, G. Badurek, M. Baron, and H. Rauch, Nature (London) 425, 45 (2003).

[40] D. A. Pushin, M. G. Huber, M. Arif, and D. G. Cory, Phys. Rev. Lett. 107, 150401 (2011).

[41] Y. Hasegawa and H. Rauch, New J. Phys. 13, 115010 (2011).

[42] J. Klepp, S. Sponar, and Y. Hasegawa, Prog. Theor. Exp. Phys. (2014) 082A01.

[43] C. W. Clark, R. Barankov, M. G. Huber, M. Arif, D. G. Cory, and D. A. Pushin, Nature (London) 525, 504 (2015).

[44] S. Basu, S. Bandyopadhyay, G. Kar, and D. Home, Phys. Lett. A 279, 281 (2001).

[45] G. Kroupa, G. Bruckner, O. Bolik, M. Zawisky, M. Hainbuchner, G. Badurek, R. J. Buchelt, A. Schricker, and H. Rauch, Nucl. Instrum. Methods Phys. Res., Sect. A 440, 604 (2000). 